\title{
Nursing Students' Hand Washing Knowledge and Attitude and their Correlation with Intention to Practice Hand Washing
}

\author{
Muyeong Seak Yang ${ }^{1}$ \\ ${ }^{1}$ Associate Professor, Nursing, Jesus university, Korea, Imedicms@jesus.ac.kr
}

\begin{abstract}
This study aims to grasp and confirm the knowledge and attitude of nursing students on hand washing, and to raise awareness of hospital infections among them. A survey was conducted to 124 nursing students in years 1-4 who are enrolled in a university located in J City, and 123 were used for final analysis. For data analysis, real, percentage, mean, standard deviation, independent t-test, and oneway ANOVA were used using the SPSS/WIN 20.0 program. As a result of the analysis, knowledge of hand washing among variables showed a significant positive correlation with attitude and intention to practice. As a result of this study, knowledge of hand washing, attitude, and intention to practice have a high correlation. Based on the results, it is suggested that the practice of hand washing can be improved by providing an educational program that improves both knowledge, attitude, and practical intentions for nursing students' hand washing.
\end{abstract}

Keywords: Hand Washing, Hand Washing Practice Intention, Hand Washing Knowledge, Hand Washing Attitude

\section{Introduction}

\subsection{The Need for Research}

Despite the efforts such as the establishment of infection control system and the development of new antibiotics, hospital related infections continue to occur. Since nurses have the most face-to-face contact with patients among all medical care personnel, they should frequently practice hand washing[1][2]. Since nurses have the most face-to-face contact with patients among all medical care personnel, they should be frequently practice hand washing. As a result of cross-infection, increased antimicrobialresistant bacteria, unnecessarily longer hospital stay, increased medical expenses, and decreased quality of medical care occur[3]. While there are various infection control methods to prevent such crossinfection, the most effective one is hand washing[4-8].

In Korea, the first case of foreign imported COVID-19 occurred in January 2020. Then, in February of the same year, centered on Daegu city and Gyeongsangbuk-do province, mass community infection occurred, and in response, the government raised the national infectious disease crisis level to serious and strengthened the 24-hour emergency response system[4]. Accordingly, national and personal interest in infection prevention and personal hygiene increased, and the necessity also emerged. Because coronavirus infectious disease (COVID19) is spread by droplets, wearing a mask and washing hands are known as the most effective methods of prevention[5]. Pathogenic or viral infections are caused by external factos as well as intrinsic factors that weakeven the immune system[6]. Hand washing is effective not only in the preventive aspect of the coronavirus, which currently causes societal problems,

Received: May 04, 2021; $1^{\text {st }}$ Review Result: June 19, 2021; $2^{\text {nd }}$ Review Result: August 12, 2021 Accepted: September 30, 2021 
but also in removing various sources of infection that can occur[7-9].

In other words, hand washing is the most fundamental, but for this reason, its importance is further emphasized and is continuously monitored and managed by medical institutions[7]. Since nurses have the most face-to-face contact with subjects among all medical care personels, they should be the most frequently exposed to hand washing. The importance of hand washing is emphasized not only in terms of the safety of the subjects but also in terms of protecting onself from the subjects. However, according to the domestic research studies, the rate of nurses practicing hand washing individually and in institutions was reported to vary from 7.7 to $52.6 \%[10]$, showing that hand washing is not thoroughly practiced.

Some previous studies suggested that nurses' awareness of the importance of hand washing was associated with increased rate of hand washing practice[11][12]. In a study targeting nursing home nurses, there was a significant relationship between the awareness level of infection control and the rate of hand washing practice. However, there was no significant relationship between nurses' knowledge level and practice rate[13]. In other previous studies, it was found that the nurses' knowledge of hand washing guidelines and social influence did not have a significant effect on the hand washing practice[14-16].

Ryu[17] said that in order to improve the hand washing education program and hand washing practice, it is necessary to examine specifically the hand washing knowledge, attitude, and practice level of nursing students who received theoretical and practical training. In a study targeting nursing students, it was pointed out that there were a large variance in hand washing knowledge and a wide range of hand washing practice score, making it difficult to say that they were practicing correct hand washing[17]. In addition, in another study conducted on nursing students, it was reported that practical training in basic infection control was not fulfilled well[18]. Furthermore, according to Park[19]'s study, it was reported that hand washing knowledge affects hand washing practice, and that correlation between hand washing knowledge and attitude and practice, including hand washing characteristics, are statistically significant. It was reported that there was a positive correlation between hand washing knowledge and attitude and practice, the higher the knowledge and the attitude, the higher the practice[20].

Given these results, it is expected that nursing students' hand washing will be greatly affected as they are exposed more frequently to hand washing through clinical practice and a new perception is formed by observing nurses in field practice. Since the developmental stage of nursing students conducting clinical practice corresponds to the early adulthood, there is a high possibility of lifestyle modifications, and in turn, it is an important period for forming desirable health habits[21]. The continuous and efficient hand washing education for nursing students who are preparing to become medical professionals is expected to have a positive effect on hand washing practice.

Hand washing is the most fundamental and essential element of COVID19 infection prevention method. Given these results, it is expected that nursing students' knowledge and attitude on hand washing will be greatly affected as they are exposed more frequently to clinical practice and a new perception is formed by observing nurses in field practice.

\subsection{The Purpose of the Study}

The purpose of this study is to find the correlation between the nursing students' knowledge and attitude and their intention to practice hand washing. The specific purposes are as follows:

First, factors affecting nursing students' hand washing are identified.

Second, the knowledge of and attitude towards hand washing, and the intention to practice hand washing are identified.

Third, the correlation between knowledge and attitude and intention to practice hand washing are identified. 


\subsection{The Research Question}

The research questions to achieve the purpose of the research are as follows:

First, does hand washing knowledge have a significant correlation with hand washing attitude?

Second, does hand washing knowledge have a significant correlation with the intention to practice hand washing?

Third, is there a significant correlation between hand washing attitude and intention to practice hand washing?

\section{Method}

\subsection{Study Design}

This study is a descriptive cross-sectional correlation research to examine the relationship between nursing students' knowledge of and attitude towards hand washing and their intention to practice hand washing. Moreover, it aims to verify the correlation with variables related to hand washing practice.

\subsection{Subjects and Data Collection}

The subjects of this study were 1st to 4th year students enrolled in the department of nursing at a university located in J city. Data was conveniently extracted and collected from October 29 to November 6, 2020. Prior to data collection, the purpose of the study was explained, and subjects who understood the purpose and agreed to participate took part in the study. Subjects were explained that data submitted in the consent form prior to participation would be used only for research purposes, processed anonymously, and kept strictly confidential. They were also told that if they no longer wished to participate during the response, they could stop at any time. The number of study subjects was calculated using the G-power 3.1 program. As a result of calculating the median effect size of .30, significant level of .05 , and power of .08 in correlation analysis, the minimum sample size required for correlation analysis was 82 . A total of 124 questionnaires were distributed in consideration of the high dropout rate, which is characteristic of web-based questionnaires. Among the responses, the study was conducted on the final 123 copies, excluding one copy that did not give an accurate response.

\subsection{Research Instrument}

\subsubsection{Hand Washing Knowledge}

In order to measure the subjects' knowledge of hand washing, six knowledge questionaires translated by Park[22] were used as a means of measuring hand washing knowledge. The scoring for each question was 1 point for 'not at all effective', 2 points for 'not very effective', 3 points for 'effective' and 4 points for 'very effective'. The scores were summed up on a 4-step Likert scale, from a minimum of 6 points to a maximum of 24 points, where the higher the average of the sum of the scores, the higher the knowledge of the hand washing effect on disease prevention. In Park[22]'s study, Cronbach's $\alpha$ was .914, whereas in this study, Cronbach's $\alpha$ was .850 .

\subsubsection{Attitude towards Hand Washing}

In order to measure the subjects' attitude towards hand washing, six attitude questionaires translated by Park[22]were used as an attitude measurement tool. The scoring for each question was 1 point for 'not at all effective', 2 points for 'not very effective', 3 points for 'effective' and 4 points for 'very effective'. The scores were summed up on a 4-step Likert scale, from a minimum of 6 points to a maximum of 24 
points, where the higher the average of the sum of the scores, the more positive the attitude towards the effect of hand washing on disease prevention. In Park[22]'s study, Cronbach's $\alpha$ was .825, whereas in this study, Cronbach's $\alpha$ was .700.

\subsubsection{Intention to Practice Hand Washing}

In order to measure the subjects' intention to practice hand washing, five intention questionaires translated by Jung[23] were used as a hand washing performance measurement tool (Whitby et al., 2006). The scoring for each question ranged from 1 point for "not at all" to 7 points for "very much". The scores were summed up on the Likert scale, from 6 to 35, where the higher the average of the sum of the scores, the stronger the intention to practice hand washing. At the time of development, Cronbach's $\alpha$ was .74. In Jung's study, Cronbach's $\alpha$ was .86, and in this study, the Cronbach's $\alpha$ was .819.

\subsection{Data Analysis}

Collected data were analyzed using the SPSS/WIN 20.0 program. The general characteristics of the subjects were analyzed using frequency and percentage. Subjects' knowledge of, attitude towards, and intention to practice hand washing were analyzed by calculating the mean and the standard deviation. The differences between the subjects in terms of their general characteristics and knowledge of, attitude towards, and intention to practice hand washing were analyzed by independent t-test and one-way ANOVA. The correlation between hand washing knowledge and attitude, and intention to practice hand washing was analyzed using Pearson's correlation coefficient.

\section{Results}

\subsection{Subjects' General Characteristics}

The gender of the subjects was $10.6 \%$ male and $89.4 \%$ female. In the case of practical training experience, $21.1 \%$ had none, $13.0 \%$ had on-campus experience, and $65.9 \%$ had on-campus and offcampus experience. Upon examining the types of people living with subjects, $59.3 \%$ were found to live with their parents, $40.7 \%$ lived alone or in a dormitory. With regards to religion, $49.6 \%$ had religion, and $50.4 \%$ had no religion[Table 1 ].

\subsection{Subjects' Hand Washing Knowledge, Attitude, and Intention to Practice Based on General}

\section{Characteristics}

There were differences in knowledge, attitude, and intention to practice hand washing depending on the general characteristics of the subjects. The hand washing knowledge was significantly different with regards to dependency status $(\mathrm{t}=-1.180, \mathrm{p}=.007)$ and religious status $(\mathrm{t}=-1.239, \mathrm{p}=0.41)$. There was no significant difference in knowledge of hand washing according to gender $(\mathrm{t}=0.080, \mathrm{p}=.937)$ and practical training experience $(\mathrm{t}=0.616, \mathrm{p}=.542)$. Attitudes toward hand washing did not show a significant difference in terms of gender $(\mathrm{t}=1.253, \mathrm{p}=.819)$, practical training experience $(\mathrm{t}=2.271, \mathrm{p}=.108)$, dependency status $(\mathrm{t}=1.638, \mathrm{p}=.306)$, and religious status $(\mathrm{t}=-.0918, \mathrm{p}=.290)$. Intention to practice hand washing was significantly high in the case of dependency status $(t=1.755, p=.042)$. There was no significant difference in intention to practice hand washing according to gender $(\mathrm{t}=-0.163, \mathrm{p}=.885)$, practical training experience $(\mathrm{t}=1.562, \mathrm{p}=.214)$, presence or absence of one's own hospital $(\mathrm{t}=3.503$, $\mathrm{p}=.064)$, and religious status $(\mathrm{t}=-1.487, \mathrm{p}=.879)$ [Table 1]. 
[Table 1] Subjects' General Characteristics and Hand Washing Knowledge, Attitude, and Intention to Practice $(\mathrm{N}=123)$

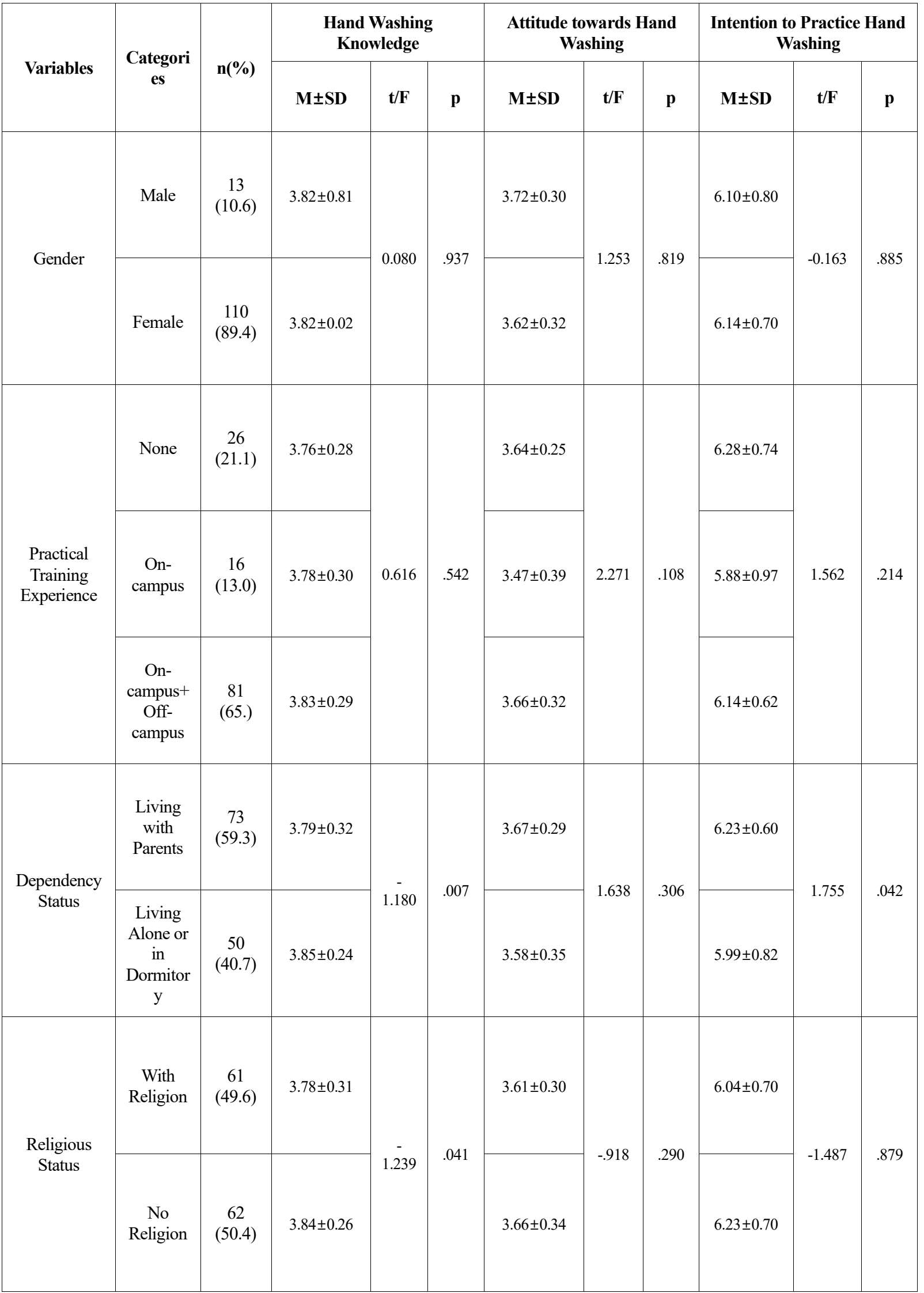




\subsection{Level of Hand Washing Knowledge, Attitude, and Intention to Practice}

Nursing students' knowledge of hand washing showed an average of $3.81 \pm 0.29$ points, and the average of hand washing attitude was $3.63 \pm 0.32$ points. The average of intention to practice hand washing was $6.13 \pm 0.71$ points [Table 2].

[Table 2] Level of Hand Washing Knowledge, Attitude, and Intention to Practice (N=123)

\begin{tabular}{|c|c|c|c|}
\hline Variables & M \pm SD & Min & Max \\
\hline Hand Washing Knowledge & $3.81 \pm 0.29$ & 3 & 4 \\
\hline Attitude towards Hand Washing & $3.63 \pm 0.32$ & 2 & 4 \\
\hline $\begin{array}{c}\text { Intention to Practice Hand } \\
\text { Washing }\end{array}$ & $6.13 \pm 0.71$ & 3 & 7 \\
\hline
\end{tabular}

\subsection{Correlation between Nursing Students' Knowledge, Attitude, and Intention to Practice Hand Washing}

Knowledge of hand washing showed statistically significant positive correlation with attitude towards hand washing $(\mathrm{r}=.369, \mathrm{p}<.001)$ and intention to practice hand washing $(\mathrm{r}=.245, \mathrm{p}<.001)$. Attitude towards hand washing showed a significant positive correlation with intention to practice hand washing $(\mathrm{r}=.369, \mathrm{p}<.001)$ [Table 3].

[Table 3] Correlation between Nursing Students' Hand Washing Knowledge, Attitude, and Intention to Practice $(\mathrm{N}=123)$

\begin{tabular}{|c|c|c|}
\hline Variables & $\begin{array}{c}\text { Knowledge } \\
\mathbf{r ( p )}\end{array}$ & $\begin{array}{c}\text { Attitude } \\
\text { r(p) }\end{array}$ \\
\hline Attitude & $.369(<.001)$ & \\
\hline Intention to Practice & $.245(<.001)$ & $.369(<.001)$ \\
\hline
\end{tabular}

\section{Discussion}

Hand washing is the most effective and safe infection control method not only for the prevention of infection in medical institutions, but also for the prevention of infectious diseases in the community and at home. Since the first case of coronavirus infection (COVID-19) in Wuhan, China in December 2019, as it has spread worldwide, the World Health Organization (WHO) declared COVID-19 a pandemic in March 2020. Accordingly, the importance of personal hygiene and hand hygiene is rising. Moreover, as medical institutions are where susceptible subjects reside, it is pertinent that nurses who have the most contact with patients in hospitals thoroughly wash their hands in order to prevent cross-infection. 
Through undergraduate courses, nursing students acquire the basic knowledge they need to have as licensed nurses after graduation. They also have the opportunity to apply nursing directly to a variety of subjects in clinical practice. If the first infection control education they encounter in an undergraduate course is conducted thoroughly at that time, then it would be possible to prevent medical infections which can occur by nursing students during practice and further perform thorough infection control as a clinical nurse. Therefore, since knowledge of and attitude towards hand washing have major effect on intention to practice hand washing, this study was conducted in an attempt to reveal the correlation between nursing students' hand washing knowledge and attitude and intention to practice hand washing. There was a statistically significant difference $(\mathrm{p}=.007)$ in the knowledge of hand washing depending on the different dependency status: whether living with parents or living alone or in a dormitory. In this study, the score for the case of living with parents was $3.81 \pm 0.29$ out of 4 points, and $3.85 \pm 0.24$ for the case of living alone or in a dormitory. The score in the case of living alone or in a dormitory was significantly higher than that in the case of living with parents. The reasons may be that this study's hand washing knowledge questionnaire was asking for a relatively simple hygiene knowledge and that the interest and knowledge level of nursing students living alone or in a dormitory regarding personal hygiene was relatively high.

Upon reviewing the differences in hand washing knowledge based on religious status, the score of hand washing knowledge was measured to be $3.78 \pm 0.31$ on a scale of 4 for having a religion and $3.84 \pm 0.26$ for having no religion. The religious status showed a statistically significant difference $(\mathrm{p}=.041)$. These results showed a different result from previous studies conducted on inpatients in one military hospital where religion was ultimately not a factor affecting hand washing knowledge[24-26]. Contrary to the results of previous studies, this study showed that the religious status had an effect on the knowledge of hand washing. Based on the fact that hand washing knowledge had a higher score with religion than without religion, hand washing knowledge can be considered to be a general knowledge that everyone has regardless of their religious status. In the future, we can suggest the need for repeated studies in order to understand the correlation between hand washing knowledge and religious status.

The score of hand washing knowledge was measured as $3.76 \pm 0.28$ in the case of no hands-on experience and $3.83 \pm 0.29$ in the case of on-campus training. According to a study by Lee et al.[17] comparing the hand washing knowledge between health science and non-health science college students, non-health science students scored $13.22 \pm 1.73$ out of 15 , and health science students scored 13.86 out of 15 . Health science students had a higher score than non-health science students. When the score of health science students is converted into a percentage, it was $92.4 \%$. The lowest score in this study with subjects having no practical training experience was converted into a percentage of $94 \%$. Compared to previous studies, this score is higher. Therefore, it is confirmed that health science students' knowledge level of hand washing is higher than non-health science students'. Since this study was conducted on college students in the nursing program, which is part of the health science department, it suggests that they all showed high scores regardless of their practical training.

In Ryu and Lim[20]'s study, hand washing attitude of nursing students prior to practical training showed a low average score of 3.42 on a scale of 5. In the study of Ko et al.[27], fourth year students who had more clinical training experience, in turn, washed hands more often compared to third year students and showed statistically significant difference. It appears that practical training has an effect on the attitude towards hand washing. However, the study results show that there was no significant difference $(p=.108)$ in the attitude towards hand washing with or without practical training, which is contrary from the results of previous studies. These results stemmed from the fact that the questionaires in this study were similar to those conducted on the general public who lack professionality. The questionaires failed to take the occupational characteristics into account. Therefore, it is possible to suggest the need for a study on hand washing attitudes with or without practical training by filling out questionaires following differing circumstances of each hospital. 
Intention to practice hand washing refers to the subject's willingness to perform hand washing. It also represents the score measured by the researcher's revised and supplemented tool, which was initially Jung[23]'s tool of intention to practice hand washing. The intention to practice hand washing showed a significant difference depending on the dependency status $(\mathrm{p}=.042)$. In the case of an independent status (living alone or in a dormitory), the intention to practice hand washing scored $5.99 \pm 0.82$ out of 7 . In the case of subjects living with parents, the score was $6.23 \pm 0.60$, which is relatively higher. It could be explained by the fact that the subjects living with parents are affected by the influence of others and have greater intention to practice hand washing and in turn wash hands more frequently[28]. When living alone, the intention to practice hand washing is low, because there is no interference by others[28]. However, results from the previous study showed that nursing students' hand washing practice had no significant correlation with the dependency status[29] $(\mathrm{p}=.910)$. Therefore, the results from this study cannot be generalized. A necessity for repeated study investigating the intention to practice hand washing under the influence of others is suggested.

\section{Conclusion and Suggestion}

This study is a descriptive cross-sectional study aimed at identifying the relationship between nursing students' knowledge, attitude, and intention to practice hand washing and verifying the correlation with variables related to hand washing practice. It is significant that the results of this study provide basic data for preparing a hand washing education program in the future by confirming that there is a high correlation between the hand washing knowledge and attitude and intention to practice hand washing.

Based on the results and discussion of this study, the following suggestions were made.

First, since this study collected data from nursing students in one particular university, it is necessary to conduct additional studies throughout an expanded area. Second, studies to observe the actual hand washing practice during nursing students' clinical practice and to analyze infection control education currently given in undergraduate courses are necessary. Third, based on the results of these previous studies, the researchers suggest a follow-up study to develop a hand washing education program, which is to increase nursing students' hand washing practice, and to verify the effect of the program.

\section{Reference}

[1] Jiyeon Park, Seung Yeon Yoo, Jae-Hoon Ko, Sangmin M. Lee, Yoon Joo Chung, Jong-Hwan Lee, Kyong Ran Peck, Jeong Jin Min, Infection prevention measures for surgical procedures during a middle east respiratory syndrome outbreak in a tertiary care hospital in South Korea, Scientific reports, (2020), Vol.10, pp.1-8, https://doi.org/10.1038/s41598-019-57216-x

[2] S. C. Kim, S. Y. Kong, G. J. Park, J. H. Lee, J. K. Lee, M. S. Lee, H. S. Han, Effectiveness of negative pressure isolation stretcher and rooms for SARS-CoV-2 nosocomial infection control and maintenance of South Korean emergency department capacity, The American Journal of Emergency Medicine, (2020), Vol.45, pp.483-489, DOI: 10.1016/j.ajem.2020.09.081

[3] A. Bianco, M. S. Capano, V. Mascaro, C. Pileggi, M. Pavia, Prospective surveillance of healthcare-associated infections and patterns of antimicrobial resistance of pathogens in an Italian intensive care unit, Antimicrobial Resistance \& Infection Control, (2018), Vol.7, No.1, pp.1-6, DOI: 10.1186/s13756-018-0337-x

[4] D. Jin, W. S. Seo, S. Lee, Arctic policy of the Republic of Korea, Ocean \& Coastal Law Journal, (2017), Vol.22, No.1, pp.85-88.

[5] J. Dolan, S. E. Dolan Looby, Determinants of nurses' use of physical restraints in surgical intensive care unit patients, American Journal of Critical Care, (2017), Vol.26, No.5, pp.373-379, DOI: 10.4037/ajcc2017244 
[6] J. L. Goldberg, Guideline Implementation: Hand Hygiene, AORN Journal, (2017), Vol.105, No.2, pp.203-212, DOI: 10.1016/j.aorn.2016.12.010

[7] KDCA, Guidelie for Hand Hygiene in Healthcare Facilities, (2014)

[8] L. Labrague, D. McEnroe-Petitte, T. Van de Mortel, A. Nasirudeen, A systematic review on hand hygiene knowledge and compliance in student nurses, International nursing review, (2018), Vol.65, No.3, pp.336-348, DOI: 10.1111/inr. 12410

[9] E. J. Lee, S. N. Kim, H. J. Park, H. M. LEE, Public Health Weekly Report, KDCA, (2019), Vol.12, No.42, pp.17571762, https://www.kdca.go.kr/board/board.es?mid=a30501000000\&bid=0031

[10] S. Jemal, Knowledge and practices of hand washing among health professionals in Dubti Referral Hospital, Dubti, Afar, Northeast Ethiopia, Advances in preventive medicine, (2018), Vol.2018, https://doi.org/10.1155/2018/5290797

[11] World Health Organization, WHO guidelines on hand hygiene in health care, Geneva: World Health Organization, (2009), http://www.who.int/gpsc/5may/tools/9789241597906/en/

[12] M. S. Lee, S. J. Hong, Y. T. Kim, Handwashing with soap and national handwashing projects in Korea: Focus on the national handwashing survey, 2006-2014, Epidemiology and Health, (2015), Vol.37, pp.1-9, DOI: https://doi.org/10.4178/epih/e2015039

[13] M. C. Freeman, M. E. Stocks, O. Cumming, A. Jeandron, J. P. T. Higgins, J. Wolf, A. Prüss-Ustün, S. Bonjour, P. R. Hunter, L. Fewtrell, V. Curtis, Hygiene and health: Systematic review of handwashing practices worldwide and update of health effects, Tropical Medicine and International Health, (2014), Vol.19, No.8, pp.906-916, https://doi.org/ 10.1111/tmi.12339

[14] T. Hovi, J. Ollgren, C. Savolainen-Kopra, Intensified hand-hygiene campaign including soap-and-water wash may prevent acute infections in office workers, as shown by a recognized exposure-adjusted analysis of a randomized trial, BMC Infectious Diseases, (2017), Vol.17, No.47, pp.1-9, https://doi.org/10.1186/s12879-016-2157-z

[15] Sun Im Han, Jungmok Kwon, Boyeong Ahn, Jaehun Ahn, Seunghun Lee, Hyun Jung Lim, HYEONJU KIM, Knowledge, Attitude and Practice (KAP) of Hand Washing for soldiers, The Journal of the Korea Contents Association, (2017), Vol.17, No.8, pp.472-482, https://doi.org/10.5392/JKCA.2017.17.08.472

[16] E. J. Yoon, M. H. Kim, Knowledge and compliance with hand hygiene by nursing students in clinical practice, Journal of Korean Academic Society of Nursing Education, (2013), Vol.19, No.3, pp.455-464, https://doi.org/10.5977/jkasne.2013.19.3.455

[17] S. M. Ryu, Y. J. Lim, Knowledge, attitude and performance on the hand washing by pre-practicum nursing students, Journal of the Korea Academia-Industrial cooperation Society, (2013), Vol.14, No.11, pp.5714-5722, https://doi.org/10.5762/kais.2013.14.11.5714

[18] K. Y. Park, Influences of perceived behavior control and self-efficacy on proper hand cleansing and hand washing practices among pre-practicum nursing students, Journal of Korean Academy of Fundamentals of Nursing, (2012), Vol.19, No.3, pp.313-321, https://doi.org/10.7739/jkafn.2012.19.3.313

[19] M. Burton, E. Cobb, P. Donachie, G. Judah, V. Curtis, W. P. Schmidt, The effect of handwashing with water or soap on bacterial contamination of hands, International Journal of Environmental Research and Public Health, (2011), Vol.8, No.1, pp.97-104, https://doi.org/10.3390/ijerph8010097

[20] N. Y. Yang, Knowledge, self efficacy and self care behavior regarding foot care among elderly diabetes mellitus patients, Journal of Korean Academy of Adult Nursing, (2009), Vol.21, No.4, pp.413-422.

[21] S. M. Ryu, Knowledge, attitude, and performance on the hand washing of health care related students, Journal of the Korea Academia-Industrial Cooperation Society, (2013), Vol.14, No.8, pp.3916-3924, https://doi.org/10.5762/ kais.2013.14.8.3916

[22] D. K. Park, M. S. Lee, B. J. Na, S. H. Bae, K. Y. Kim, C. W. Kim, E. Y. Kim, K. Y. Kim, Knowledge, attitude, and practice of handwashing in high school students, Journal of Korean Society Maternal and Child Health, (2008), Vol.12, No.1, pp.74-91, DOI : 10.21896/jksmch.2008.12.1.74

[23] I. Ajzen, Attitudes, personality, and behavior, (2nd Edition), Open University Press, (2005) 
[24] E. A. Jenner, P. W. B. Watson, L. Miller, F. Jones, G. M. Scott, Explaining hand hygiene practice: An extended application of the theory of planned behaviour, Psychology, Health and Medicine, (2002), Vol.7, No.3, pp.311-326, https://doi.org/10.1080/13548500220139412

[25] M. R. Lee, H. Y. So, M. K. Cho, Determinants of nurses' handwashing practice, Korean Journal of Adult Nursing, (2005), Vol.17, No.2, pp.268- 275, UCI : G704-000678.2005.17.2.014

[26] S. Y. Jeong, O. S. Kim, The structural model of hand hygiene behavior for the prevention of healthcare-associated infection in hospital nurses, Korean Journal of Adult Nursing, (2012), Vol.24, No.2, pp.119-129, https://doi.org/10.7475/kjan.2012.24.2.119

[27] D. E. Montano, D. Kasprzyk, Theory of reasoned action, theory of planned behavior, and the integrated behavioral model, (Health behavior: Theory, research and practice), John Wiley \& Sons, (2008), pp.95-124.

[28] S. M. Ryu, S. Y. Kang, Effect of an Infection Prevention Education Program on Knowledge of infection and Hand washing Performance for Nursing Students, Health Service Management Review, (2010), Vol.4, No.2, pp.31-37, DOI : 10.18014/hsmr.2010.4.2.31

[29] E. H. Choi, I. S. Jang, J. Y. Choi, The effect of an educational hand washing program on knowledge, attitude and performance of hand washing in undergraduates, Journal of the Korean Society of School Health, (2014), Vol.27, No.1, pp.39-49, https://doi.org/10.15434/kssh.2014.27.1.039 